\title{
Atendimento odontológico do paciente com diabetes melito: recomendações para a prática clínica
}

\author{
Crésio Alves ${ }^{1}$ \\ Márcia Brandão ${ }^{2}$ \\ Juliana Andion ${ }^{3}$ \\ Rafaela M enezes ${ }^{3}$ \\ Fabíola Carvalho ${ }^{3}$
}

\begin{abstract}
Resumo
D iabetes melito (D M ) abrangeum grupo dedistúrbios metabólicosquetêm em comum a presença dehiperglicemia. Além das manifestações sistêmicas, o D M mal controlado apresenta importantes repercussões sobrea saúdeoral (eg., doença periodontal, xerostomia, hiposalivação, susceptibilidadea infecções, dificuldade decicatrização). Estetrabalho tem por objetivo destacar a importância do conhecimento básico do odontologista sobreD M epropor um protocolo de aten dimento dentário para esses pacientes. A revisão bibliográfica foi realizada nos bancos de dados M ED LIN E e LILACS, com pesquisa em artigos publicados nos últimos dez anos, a partir das palavras-chave: diabetes mellitus, dentistry, oral health e periodontal disease. O sresultados são estruturados em tópicos. N a primeira parte, são atualizadas informações sobre diagnóstico, manifestações clínicas, repercussões orais e tratamento do D M . N a segunda parte, propomos um protocolo de atendimento, no qual são discutidas, de modo didático (e.g., anamnese, examefísico, exames complementares, conduta), as dúvidas mais comuns em relação à consulta odontológica do diabético (e.g., profilaxia antibiótica em procedimentos com risco debacteremia, uso deantiinflamatórios, sedativos eanestésicos com vasoconstrictores, tratamento paliativo versus definitivo, como proceder em casos de hipoglicemia ehiperglicemia, quando suspeitar de um D M não diagnosticado). Ao final, uma ficha clínica sumariza os principais aspectos da consulta odontológica do pacientediabético. Conclui-se quediabéticosbem controlados esem complicações podem ser tratados demodo similar a não-diabéticos, para a mai oria dos procedimentos de rotina. 0 cirurgião-dentista, em comunicação com o médico assistente, desempenha um importantepapel na promoção emanutenção do bem-estar equalidade de vida do paciente diabético.
\end{abstract}

Palavras-chaves: D iabetes melito - 0 dontologia; saúdeoral; doença periodontal.

\section{INT RO DUÇÃO}

D iabetes melito (D M) representa um grupo de doenças caracterizadas por hiperglicemia, como resultado da deficiência na pro- dução de insulina, resistência periférica à sua ação, ou ambas. A incidência do D M vem atingindo proporções epidêmicas. De acordo com

\footnotetext{
1 Professor Assistente de Pediatria. Faculdade de M edicina. Universidade Federal da Bahia - UFBA. Salvador - BA. Coordenador da Residência em Endocrinologia Pediátrica. H ospital Universitário Professor Edgard Santos. Universidade Federal da Bahia - UFBA. Salvador - BA

2 Professora Assistente de 0 dontologia Social. Fundação Baiana para o D esenvolvimento das Ciências - FBD C. Salvador - BA

3 Acadêmico de 0 dontologia Faculdade de O dontologia - UFBA. Salvador - BA
}

Correspondência para / Correspondence to:

Crésio Alves

Faculdade de Medicina - UFBA

Rua Plínio M oscoso, 222/601

40.157-190. Salvador - BA - Brasil

Tel.: (71) 9975-8220

E-mail: cresio.alves@uol.com.br 
a $O$ rganização M undial de Saúde, no ano de 2030, 300 milhões de pessoas serão diabéticas (EISELEIN; SCH WARTZ; RUTLEDGE, 2004). N o Brasil, aproximadamente de 8 a 10 milhões de pessoas são portadores de DM .

Em longo prazo, a hiperglicemia pode causar disfunção e falência de diversos órgãos. D entre as complicações sistêmicas, incluem-se as alterações bucais, com destaque para a doença periodontal, considerada por alguns como a sexta complicação crônica do D M (SO UZA et al., 2003; O RSO; PAN GN ON CELLI, 2002). Estima-se que 3 a $4 \%$ dos pacientes adultos que se submetem a tratamento odontológico são diabéticos, e uma parte significante deles desconhece ter a doença (SO U ZA et al., 2003).

D evido à alta prevalência, à morbidade, às manifestações bucais e às dúvidas sobreo atendimento odontológico dos indivíduos com D M , este artigo tem por objetivo atualizar o conhecimento básico sobre esse assunto e propor um protocolo de atendimento preventivo e terapêutico que 0 auxilie o odontólogo quando da avaliação desses pacientes.

\section{METODOLOGIA}

Foi realizada pesquisa bibliográfica nos bancos de dados MEDLINE, LILACS e
PU BM ED, para seleção de artigos publicados nos últimos 10 anos, sob a forma de consenso, trabalhos originais e de revisão, a partir das seguintes palavras-chaves: 1 . diabetes mellitus; 2 . dentistry; 3. oral health; e 4. periodontal disease.

\section{DIABETES MELITO: NOÇÕES GERAIS}

\section{D iagnóstico e manifestações clínicas}

Em indivíduos normais, a concentração plasmática de glicose situa-se entre 70 e $99 \mathrm{mg} /$ $\mathrm{dL}$. N íveis superiores indicam graus variados de tolerância à glicose (pré-diabetes) ou diabetes, cujos critérios diagnósticos foram recentemente atualizados pela American Diabetes Association (TABELA 1). As duas formas mais comuns de DM são: diabetes melito tipo 1 (DM 1) e diabetes melito tipo 2 (DM 2) (AMERICAN DIABETES ASSOCIATION, 2004). $O$ utras formas menos comuns são os diabetes gestacionais, diabetes associados a medicamentos (corticóides), doenças do pâncreas (fibrose cística), infecções (rubéola congênita) e síndromes genéticas.

O DM 1 é causado pela interação de fatores genéticos e ambientais, levando à destruição auto-imune das células â-pancreáticas produtoras de insulina (M ISTRO et al., 2003; AN TUNES et al., 2003). $O$ DM 1 apresenta

Tabela 1. Critérios para o diagnóstico do diabetes

\begin{tabular}{l|l|c|c|c}
\hline \multirow{2}{*}{ Categoria } & \multicolumn{3}{c}{ Glicemia $(\mathrm{mg} / \mathrm{dL})$} \\
\cline { 3 - 5 } \multicolumn{2}{l}{ Glicemia normal } & Jejum & Ao acaso & $2 \mathrm{~h}(\mathrm{TTOG})$ \\
\hline \multirow{3}{*}{ Pré-diabetes } & $\begin{array}{l}\text { Glicemia de } \\
\text { jejum alterada }\end{array}$ & $100-125$ & - & $<140$ \\
\cline { 2 - 5 } & $\begin{array}{l}\text { Intolerância a } \\
\text { glicose }\end{array}$ & -- & - & $\geq 140 \mathrm{e}<200$ \\
\hline \multicolumn{2}{l}{ Diabetes tipo 1 ou 2 } & $\geq 126$ & - & $\geq 200$ \\
\hline \multicolumn{2}{l}{ Diabetes gestacional } & $\geq 110$ & $\geq 200$ & $\geq 140$ \\
\hline
\end{tabular}

Fonte: AMERICAN DIABETES ASSO CIATION, 2004.

N otas:- Glicemia de jejum: ausência de ingestão calórica por pelo menos 8 horas.

- Glicemia ao acaso: glicemia realizada em qualquer hora do dia sem levar em consideração o tempo desde a última refeição. 0 diagnóstico deve ser confirmado medindo a glicemia em jejum ou 2 h após O TTO G.

- TTOG (Teste de Tolerância O ral a Glicose): é a medida da glicemia basal e 2 horas após a ingestão de 1,75 g/ $/ \mathrm{gg}$ de glicose (máx. $75 \mathrm{~g}$ ).

- U m resultado positivo em qualquer dos testes acima deverá ser confirmado nos dias subseqüentes medindo a glicemia em jejum ou 2 h após o TTOG. 
dois picos de incidência: entre 5-7 anos e na puberdade. N ão há predileção por gênero. Essa patologia acomete 5 a $10 \%$ dos pacientes com D M . Q uando o quadro clínico se inicia, já houve perda de 80 a $90 \%$ da capacidade de produção de insulina (M AN N A et al., 2002). As manifestações clássicas incluem poliúria (aumento do volume urinário, devido à diurese osmótica, causada pelo excesso de glicose), polidipsia (aumento da sede para compensar a perda de água pela urina), polifagia (aumento da fome, para compensar o estado catabólico resultante da deficiência de insulina) e perda de peso. M enos freqüentemente, alguns pacientes são diagnosticados em cetoacidose diabé tica.

O DM 2 decorre da combinação de deficiência relativa de insulina e resistência insulínica (FERREIRA; VAN N U CCI, 2004). Essa é a forma mais comum da doença, responsável por $90-95 \%$ dos casos. Geralmente é associada à obesidade, hipertensão e dislipidemia, acometendo principalmente indivíduos com mais de 40 anos (MOORE et al., 2001; ANTUNES et al., 2003). Um número significativo de pessoas com DM 2 é assintomática ou oligossintomática. I sso faz com que o diagnóstico seja tardio e muitas delas já apresentem complicações microvasculares ou macrovasculares quando do diagnóstico.

\section{M onitoração e tratamento}

O DM não tem cura. A abordagem terapêutica inclui: dieta, atividade física, educação, apoio psicossocial e medicamentos para controle da hiperglicemia. A insulinoterapia é indicada para todos os pacientes com D M 1 e alguns com D M 2. O s hipoglicemiantes orais são indicados para o DM2 (CALLIARI, 2004). $\mathrm{Na}$ insulinoterapia, através de injeções subcutâneas, utiliza-se insulina humana de ação intermediária (N PH ) e ação rápida (Regular); e, mais recentemente, os novos análogos de ação lenta (Glargina [Lantus ${ }^{\circledR}$ ], D etemir [Levemir ${ }^{\circledR}$ ]) e ultra-rápida (Lispro [H umalog $®$ ], A spart [N ovoRapid $®]$ ). Uma pequena proporção de indivíduos ainda faz uso de insulina de origem animal. A partir do final de 2006, estará disponível, para uso clínico, a primeira insulina inalatória (Exubera $\left.{ }^{\circledR}\right)$. O s hipoglicemiantes orais podem ser classificados em sulfoniuréias e meglitinidas (estimulam a produção de insulina), biguanidas (diminuem a resistência à insulina), glitazonas (aumentam a ação da insulina) e inibidores da alfa-glicosidase (diminuem a absorção do açúcar).

Para que se consiga melhor resultado, o paciente e sua família devem ser orientados quanto à importância do tratamento. A automonitoração domiciliar da glicemia permite o conhecimento das variações gl icêmicas durante o dia, levando a decisões terapêuticas mais acertadas (M EALEY et al., 1999). A avaliação trimestral da hemoglobina glicosilada (H bA1c) é o padrão-ouro para a monitoração da glicemia. Ela medea ligação da glicose com a hemoglobina, no interior das hemácias. Como as hemácias têm uma vida média de 3 meses, a $\mathrm{H}$ bAlc reflete a média de controle glicêmico nesse período. 0 valor normal para indivíduos adultos é $<7 \%$. Para crianças, os valores recomendados são: préescolares $<8,5 \%$, escolares $<8 \%$ e adolescentes $<7,5 \%$ (SILVERSTEIN et al., 2005).

\section{Complicações}

As principais complicações crônicas do DM são nefropatia, retinopatia, neuropatia e doença macrovascular. As complicações agudas, hipoglicemia e hiperglicemia, são discutidas a seguir:

\section{H ipoglicemia}

- D efinição: glicemia < 70 mg/dL.

- Causas: dose excessiva de insulina ou hipoglicemiantes orais, omissão ou atraso das refeições, ingestão de álcool, exercícios prolongados, insuficiência renal, má absorção intestinal, drogas com ação hipoglicemiante (antiinflamatórios não-hormonais).

- Sintomas: adrenérgicos (tremor, sudorese, palidez, taquicardia, pal pitação, fome) e neuroglicopênicos (cefaléia, tontura, sonolência, irritabilidade, fraqueza, confusão mental, visão turva, incoordenação motora, desmaio, convulsão e coma).

- Classificação: assintomática, sintomática leve (o paciente pode tratar sua hipoglicemia) e sintomática grave (o paciente 
requer ajuda de outra pessoa para tratar a hipoglicemia).

- D iagnóstico: a suspeita de hipoglicemia deve ser confirmada medindo-se a glicemia capilar. C aso isso não seja possível, o paciente sintomático deverá ser tratado como se apresentasse uma verdadeira hipoglicemia.

- Tratamento: o tratamento depende da gravidade. 0 paciente consciente deverá inge rir o equivalente a 15 gramas de glicose: 1 comprimido de glicose, 1 sachê de mel ou açúcar, 1 copo de suco de frutas ou de refrigerante, 1 coIher de sopa rasa de açúcar, 2 balas de mel ou 3 colheres de geléia. $\mathrm{N}$ a falta desses, ingerir qualquer outro alimento com açúcar. Em seguida, monitorar a glicemia capilar a cada 15 minutos, até a sua normalização. 0 paciente inconsciente não deverá receber nada por via oral, com exceção de um pouco de açúcar aplicado na bochecha. 0 tratamento ideal para esses casos é a administração de glicose a $10 \%$ por via endovenosa (em média 30-50 mL).

\section{H iperglicemia}

- D efinição: em diabéticos, glicemia > 140-180 mg/dL, a depender do grau de controle desejado.

- Causas: omissão ou subdose de insulina ou hipoglicemiantes orais, excessos alimentares, medicamentos que aumentam a glicemia (corticóides), infecções, cirurgia, estresse físico, traumático, metabólico ou emocional.

- Sintomas: poliúria, polidipsia, polifagia, perda de peso, hálito cetônico (odor de maçã ou fruta passada, na respiração). Se não controlada, a hiperglicemia pode evoluir para cetoacidose diabética, que se caracteriza por taquicardia, hipotensão, náusea, vômito, dor abdominal, desidratação (mucosa oral seca, saliva espessa), respiração de Kussmaul (respiração rápida e profunda), alteração do sensório, choque e coma.

- Classificação: casos leves (poliúria, polidipsia, polifagia) e casos graves (cetoacidose diabética).

- Diagnóstico: a suspeita de hiperglicemia deve ser confirmada pela medida da glicemia capilar.
- Tratamento: nos casos leves, o paciente fará os ajustes de doses orientados por seu mé dico. $\mathrm{N}$ a hiperglicemia grave, o paciente deverá ser encaminhado para uma U nidade de Emergência.

\section{M anifestações bucais}

As manifestações bucais observadas no paciente com D M , embora não específicas dessa doença, têm sua incidência ou progressão favorecida pelo descontrole glicêmico. Os distúrbios da cavidade bucal mais freqüentes nos diabéticos são: xerostomia, hiposalivação, síndrome de ardência bucal, glossodinia, distúrbios da gustação, infecções, ulcerações na mucosa bucal, hipocal cificação do esmalte, perda precoce de dentes, dificuldade de cicatrização, doença periodontal, hálito cetônico e líquen plano (SOUZA et al., 2003; VERNILLO, 2003).

Existe controvérsia sobre a associação entre diabetes e cáries (VERN ILLO, 2003). Se, por um lado, poder-seia supor maior susceptibilidade à cárie entre diabéticos (maior concentração de glicose salivar, aumento da acidez do meio bucal, aumento da viscosidade e diminuição do fluxo salivar, hipocalcificação do esmalte, distúrbios salivares e periodontais), outros fatores, como menor ingestão de sacarose, poderiam contribuir para diminuir sua ocorrência (SO U ZA et al., 2003; AM ARAL; RAM OS; FERREIRA, 2006). Alguns autores não encontraram relação entre essas duas doenças (M IRALLES et al., 2002), enquanto outros relatam que pacientes com controle metabólico inadequado apresentam piores resultados nos índices CPO -D (D ente C ariado/Perdido/O bturado) (FERREIRA; VAN N U CCI, 2004).

A produção e o fluxo salivar são mediados pelo sistema nervoso autônomo, através da ação do neurotransmissor colinérgico acetilcolina. A xerostomia (sensação subjetiva de boca seca que, geralmente, mas não necessariamente, está associada com a diminuição da quantidade de saliva) é relatada por 10 a 30\% dos diabéticos (N EVILLE et al., 2004; COSTA et al., 2004; TÓFOLI et al., 2005). A hipossalivação pode causar glossodinia, úlceras, 
queilites, língua fissurada, lesões cariosas e dificuldade de retenção das próteses, com trauma dos tecidos moles, o que predispõe a infecções (VERN ILLO , 2003). Ela tende a se agravar em fases de descontrole metabólico, pelo fato de a desidratação aumentar os gradientes osmóticos dos vasos sanguíneos em relação às glândulas salivares, limitando a secreção de saliva (M O ORE et al., 2001). 0 uso de drogas com ação anticolinérgica é outra condição que leva à redução da produção e do fluxo salivar. O s principais medicamentos com efeitos anticolinérgicos são: antidepressivos (amitriptilina $\left[\right.$ Amytril ${ }^{\circledR}$, Tryptanol $\left.®\right]$, sertralina [Zoloft $\left.®\right]$, paroxetina $[$ Atropax $®])$, anti-histamínicos (loratadina [C laritin ${ }^{\circledR}$, Loralerg ${ }^{\circledR}$ ], prometazina [Fenergan $\left.{ }^{\circledR}\right]$ ]) e derivados benzodiazepínicos (alprazolam [Frontal ${ }^{\circledR}$ ], diazepan [Valium ${ }^{\circledR}$, D iempax ${ }^{\circledR}$ ], Iorazepan [Lorax ${ }^{\circledR}$ ]) (WYN N ; MEILLER, 2001).

Indivíduos com DM estão mais propensos a desenvolver infecções e abscessos na cavidade bucal, o que pode agravar o controle me tabólico. A susceptibilidade para infecções orais, a exemplo da candidíase oral, é favorecida pela hiperglicemia, diminuição do fluxo salivar e alterações na composição da saliva, através de modificações em proteínas antimicrobianas como lactoferrina, lizozima e lactoperoxidase (VERN ILLO, 2003; TEKELI et al., 2004).

A susceptibilidade e a progressão da doença periodontal, no paciente diabético, está associada ao descontrole metabólico, à presença de complicações, ao espessamento dos vasos sanguíneos, à redução da quimiotaxia dos neutrófilos, à glicosilação (ligação de glicose a proteínas) de proteínas estruturais, formando produtos avançados de glicosilação (AGEs), função reduzida dos fibroblastos e alterações gené ticas, como herança de determinados antígenos de histocompatibilidade (SO UZA et al., 2003; ANTUNES et al., 2003; SO UTHERLAND et al., 2006). Um estudo mostrou prevalência de 9,8\% de doença periodontal em pacientes com DM 1, quando comparados a 1,6\% em não diabéticos (CIAN CO LA et al., 1982). N o D M 2, o risco de doença periodontal é três vezes maior do que na população em geral (VERNILLO,
2003). A manifestação inicial é gengivite (sangramento, recessão gengival), que, se não cuidada, progride para doença periodontal severa, com formação de bolsas periodontais ativas, abscessos periodontais, osteoporose trabecular e destruição do suporte periodontal (SOUZA et al., 2003; PALMER; SOORY, 2005).

Algumas hipóteses consideram uma associação bidirecional entre diabetes e doença periodontal. $\mathrm{N}$ essas hipóteses, o diabetes alteraria a resposta imunológica e metabólica do organismo, favorecendo e exacerbando a doença periodontal, e ela contribuiria para o mau controle dos níveis glicêmicos (WEH BA; RODRIGUES; SOARES, 2004). Assim, a manutenção da saúde dos tecidos periodontais contribui para um melhor controle metabólico, reduzindo as necessidades de insulina e os níveis de hemoglobina glicosilada (KAWAM U RA, 2002).

\section{DIABETES MELITO: PROTOCOLO DE ATENDIMENTO ODONTOLÓGICO}

$N$ as consultas eletivas, é útil obter um relatório do médico assistente, enquanto nas consultas de emergência, pode ser necessário um contato telefônico com o mesmo. Lembrar que, muitas vezes, o problema dentário é a causa de descompensação do $D M$ e que seu tratamento pode ser a única maneira de restaurar controle metabólico.

\section{Anamnese}

Grande parte dos pacientes com DM 2 desconhece a sua doença. Portanto, o cirurgiãodentista deve estar atento para suspeitar dos casos não diagnosticados, encaminhando para o mé dico assistente aqueles indivíduos que apresentem sintomatologia oral (candidíase, xerostomia) ou sistêmica sugestiva de DM 1 (poliúria, polidispsia, polifagia, perda de peso) ou DM 2 (obesidade, dislipidemia, hipertensão).

$\mathrm{N}$ os pacientes com diagnóstico prévio, 0 dentista deve se informar sobre o tipo da doença (D M 1, D M 2,), duração da enfermidade, 
terapia (dieta, insulina, hipoglicemiantes, horário da última dose desses medicamentos), horário da última refeição, controle metabólico (H bA1c), complicações (nefropatia, neuropatia, retinopatia), sintomas de hipoglicemia, história de hospitalização e cetoacidose, infecções sistêmicas (febre, mal estar, uso de antibióticos, antiinflamatórios e analgésicos) e medicamentos para tratar complicações associadas ao DM. O s antihipertensivos podem causar desidratação, os antidepressivos e benzodiazepínicos podem levar àhiposalivação, os antiinflamatórios não-esteróides (AIN ES) podem potencializar os efeitos dos hipoglicemiantes orais, aumentando o risco de hipoglicemia, e os corticóides podem agravar a hiperglicemia (TÓ FO LI et al., 2005; SO UZA et al., 2003). Também é importante questionar sobre hipertensão arterial, uso de álcool (causa de hipoglicemia) e tabagismo. 0 médico assistente deverá ser consultado no caso de pacientes com complicações crônicas, para discutir possíveis modificações no plano terapêutico, principalmente se procedimentos cirúrgicos forem necessários (VERN ILLO et al., 2003). $N$ as diabéticas grávidas, é fundamental se informar dos cuidados em relação ao uso de medicamentos e exposição aos raios $X$. Em relação à saúde bucal, devem ser feitas perguntas sobre higiene oral (freqüência de escovação, uso de fio dental, visitas regulares ao dentista), sintomatologia sugestiva de manifestações bucais associadas ao D M (sangramento ou pus na gengiva, dentes "moles ou bambos" excluindo a troca de dentição decídua, candidíase oral, abscessos) e tratamento periodontal prévio.

\section{Exame físico}

0 exame físico da cavidade oral deve se guir a rotina habitual. No exame supragengival, enfatizar a avaliação de sangramento gengival (gengivite), placa bacteriana e possíveis fatores retentivos (cálculo dental, cárie, próteses maladaptadas, restaurações com falta ou excesso de material restaurador, raízes residuais, posicionamento dental atípico, hiperplasias gengivais e presença de aparelhos ortodônticos). A avaliação das áreas subgengivais deve observar a profundidade de sondagem, sinais clínicos de in- flamação (sangramento ou supuração à sondagem), níveis de inserção periodontal e recessão gengival. $N$ ão esquecer de investigar lesões de furca, grau de mobilidade das unidades dentais, hálito cetônico, infecções bucais e hiposalivação. $0 \mathrm{~s}$ pacientes com nefropatia diabética ou hipertensão arterial devem ter sua pressão arterial aferida antes do inicio dos procedimentos odontológicos.

\section{Exames complementares}

$\mathrm{N}$ aqueles onde a história clínica sugerir DM 1 ou DM 2, solicitar exames laboratoriais que comprovem o diagnóstico e, caso esse seja confirmado, encaminhar o paciente para 0 endocrinologista (SO UZA et al., 2003).

Aqueles com diagnóstico prévio de DM deverão ter sua glicemia capilar avaliada antes do inicio do procedimento, para identificar e tratar uma possível hipoglicemia ou hiperglicemia (MISTRO et al., 2003).

Radiografias de unidades dentárias específicas e radiografias panorâmicas estão indicadas para complementar o exame físico, quando da avaliação de doença periodontal, inserção óssea, tratamentos endodônticos, abscessos, lesões cariosas e comprometimento ósseo (SAN CH ES et al., 2004).

\section{Aspectos importantes da consulta odontológica do paciente diabético}

- Controle metabólico: pacientes bem controlados, sem complicações crônicas, com boa higiene bucal e acompanhamento médico regular podem ser tratados sem necessidade de cuidados especiais, uma vez que eles respondem de forma favorável e da mesma forma que não-diabéticos (SO UZA et al., 2003). N aqueles com descompensação metabólica e (ou) múltiplas complicações, 0 tratamento odontológico será paliativo e indicado em situações de urgência, como presença de dor e infecções. A terapia definitiva será adiada até estabilização das condições metabólicas (T Ó FO LI et al., 2005). Pacientes com glicemia, em jejum, acima de $230 \mathrm{mg} / \mathrm{dL}$, têm um aumento de $80 \%$ no risco de desenvolver infecção (SH IP, 2003).

- J ejum e horário do atendimento: no dia 
que precede a consulta odontológica, a dieta será de acordo com o padrão usado pelo paciente. Em caso de necessidade de jejum prolongado, ou antecipação de redução na ingestão alimentar após o procedimento, poderá ser necessária a redução ou a omissão de doses dos hipoglicemiantes orais ou da insulina (MEALEY et al., 1999; SOUZA et al., 2003). 0 jejum desnecessário é um dos fatores precipitantes para hipoglicemia. 0 atendimento odontológico pode ser feito em qualquer horário do dia (SO UZA et al., 2003; HORLIAN A et al., 2005).

- Equipamento especial: é importante que o cirurgião-dentista possua um glicosímetro para checar a glicemia capilar antes da consulta ou durante a mesma, caso se suspeite de hipoglicemia ou hiperglicemia.

- Ajustes nas doses de insulina e hipoglicemiantes orais: ajustes nas doses de medicamentos estão indicados quando houver necessidade de jejum prolongado, uso de AINES ou corticóides, realização de procedimentos invasivos e antecipação de dor ou estresse importante. O s hipoglicemiantes orais podem ter seu efeito potencializado pelo uso concomitante de AINES, que deslocam os hipoglicemiantes do seu sítio de ligação, aumentando o efeito hipoglicemiante, enquanto os corticosteróides têm efeito hiperglicemiante. Assim, a prescrição desses dois medicamentos deve ser criteriosa, sendo necessário entrar em contato com o médico responsável, para ajustar sua posologia (TÓFOLI et al., 2005; SAN CHES et al., 2004). A não ser nessas situações, os pacientes devem ser instruídos a manter a dose usual de seus medicamentos.

- Ajustes na monitoração domiciliar da glicemia: a glicemia capilar deverá ser checada mais freqüentemente nos primeiros dias após a realização de procedimentos dentários demorados, traumáticos ou estressantes, ou quando for necessário uso de antibióticos, AINES, corticóides ou restrição alimentar.

- Ansiedade e medo: esses sintomas devem ser controlados, pois levam à liberação de adrenalina, causando aumento na glicemia (TÓ FOLI et al., 2005). Visando a reduzir essa tensão, recomenda-se utilizar técnicas de sedação auxiliar, quando apropriadas. A sedação inalatória é relatada por alguns autores como a técnica mais segura e previsível de sedação consciente (TÓ FOLI et al., 2005; HORLIANA et al., 2005). O s benzodiazepínicos usados para tratar ansiedade (diazepan, lorazepan), embora possam causar hiposalivação, não são contraindicados.

- Dor: a dor pode ser controlada com analgésicos simples (acetaminofeno, dipirona) e AIN ES (nimesulida, ibuprofeno, diclofenaco). $\mathrm{N}$ os casos graves, usar preparações com codeína.

- Inflamação: a inflamação pode ser controlada com AIN ES. Evitar corticóides pelo risco de hiperglicemia. 0 uso de clorexidina durante o tratamento odontológico é recomendado para controle da placa, manutenção de flora não patogênica e prevenção da doença periodontal severa (SAN CHES et al., 2004).

- Antimicrobianos: a terapêutica antimicrobiana para pacientes com bom controle glicêmico é semelhante ao de não-diabéticos, ou seja, só deve ser realizada quando existirem sinais e sintomas sistêmicos de infecção (TÓ FOLI et al., 2005). N os pacientes com doença mal controlada, mesmo na ausência de sinais e infecção, preconiza-se profilaxia antibiótica nos procedimentos que geram bacteremia importante (QUADRO 1) (DAJAN I et al, 1997). Caso seja necessária a prescrição curativa, podem ser usados: penicilinas (amoxicilina, ampicilina), cefalosporinas (cefal exina) ou macrolídeos (azitromicina, claritromicina) (TÓ FOLI et al., 2005). N ão se preocupar em prescrever antibióticos sob a forma de suspensão oral com receio de agravar a hiperglicemia. N essas situações, o paciente estará checando sua glicemia capilar mais vezes e poderá usar insulina de ação rápida ou ultra-rápida, caso a glicemia ultrapasse um determinado valor estabelecido pelo médico assistente.

- Anestesia local: não existe consenso sobre o tipo de anestésico local a ser usado no tratamento odontológico do diabético. Em pacientes compensados, os anestésicos locais com adrenalina ou noradrenalina podem ser usados sem problemas (TÓ FOLI et al., 2005). O s anestésicos de longa duração não constituem a melhor escolha, porque têm influência no 


\begin{tabular}{|l|l|l|}
\hline \multicolumn{1}{|c|}{ Situação } & \multicolumn{1}{|c|}{ Antibiótico } & \multicolumn{1}{c|}{ Esquema } \\
\hline Regime padrão & Amoxacilina & $\begin{array}{l}\text { Adultos: } 2 \text { gramas; crianças } 50 \mathrm{mg} / \mathrm{Kg}, \mathrm{VO}, 1 \\
\text { hora an tes do procedimento } \\
\text { Adultos: } 2 \text { gramas; crianças } 50 \mathrm{mg} / \mathrm{Kg}, \mathrm{IM} \text { ou } \\
\text { EV, } 30 \text { minutos antes do procedimento }\end{array}$ \\
\hline $\begin{array}{l}\text { Alergia a Penicilina } \\
\text { (VO é possível) }\end{array}$ & $\begin{array}{l}\text { Azitromicina ou } \\
\text { Claritromicina } \\
\text { Cefalexina ou } \\
\text { Cefadroxil } \\
\text { Clindamicina }\end{array}$ & $\begin{array}{l}\text { Adultos: } 500 \mathrm{mg} \text { crianças } 15 \mathrm{mg} / \mathrm{Kg}, \mathrm{VO}, \\
\text { Adultos: } 2 \text { gramas; crianças } 50 \mathrm{mg} / \mathrm{Kg}, \mathrm{VO}, 1 \\
\text { hora antes do procedimento } \\
\text { Adultos: } 600 \mathrm{mg} \text { crianças } 20 \mathrm{mg} / \mathrm{Kg}, \mathrm{VO}, \\
1 \text { hora antes do procedimento }\end{array}$ \\
\hline $\begin{array}{l}\text { Alergia a Penicilina } \\
\text { (VO é impossível) }\end{array}$ & $\begin{array}{l}\text { Adultos: } 1 \text { grama; crianças } 25 \mathrm{mg} / \mathrm{Kg}, \mathrm{IM} \text { out } \\
\text { EV, } 30 \text { minutos antes do procedimento } \\
\text { Cefalina ou } \\
\text { Clultos: } 600 \mathrm{mg} \text {; crianças } 20 \mathrm{mg} / \mathrm{Kg}, \mathrm{EV}, 30 \\
\text { minutos antes do procedimento. }\end{array}$ \\
\hline
\end{tabular}

Q uadro 1- Antibioticoterapia profilática para procedimentos dentários.

\begin{tabular}{|c|c|}
\hline Patologia bucal & Tratamento \\
\hline Xerostomia e hiposalivação & $\begin{array}{l}\text { - Enxaguatórios bucais; estimulantes da saliva (gomas de } \\
\text { mascar sem açúcar); controle glicêmico; reduzir dose ou } \\
\text { suspender uso de antidepressivos tricíclicos (causam } \\
\text { ressecamento da boca) em consulta com médico assistente. }\end{array}$ \\
\hline Síndrome da ardência bucal & $\begin{array}{l}\text { - Tratamento da xerostomia e hipossalivação; controle } \\
\text { glicêmico; e nos casos severos: baixas doses de } \\
\text { benzodiazepínicos ou antidepressivos tricíclicos prescritos } \\
\text { pelo médico assistente. }\end{array}$ \\
\hline $\begin{array}{l}\text { Infecção recorrente pelo vírus } \\
\text { Herpes simplex }\end{array}$ & $\begin{array}{l}\text { - Aciclovir (Zovirax }(\text {, creme } 5 \% \text {, comp. } 200 \text { e } 400 \mathrm{mg} \text { ). } \\
\text { Uso: tópico }(1 \text { aplicaçáo } 5 \mathrm{x} / \mathrm{dia}) \text { ou oral }(<2 \text { anos }=100 \\
\text { mg e }>2 \text { anos }=200 \mathrm{mg}, 5 \mathrm{x} / \mathrm{dia}) \text { por } 5-10 \text { dias. Ajustar } \\
\text { doses no paciente com insuficiência renal. }\end{array}$ \\
\hline Infecção por Cândida albicans & 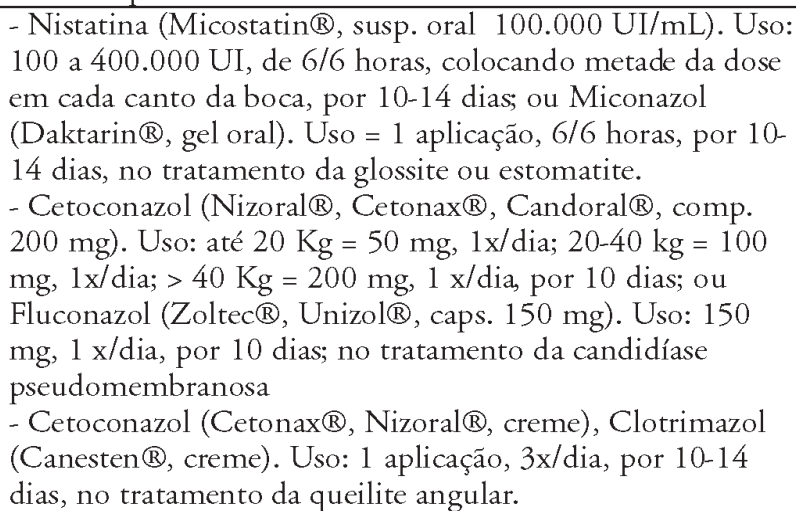 \\
\hline Doença periodontal & $\begin{array}{l}\text { Tratamento náo cirúrgico (raspagem, alisamento radicular, } \\
\text { controle de placa, higiene oral, bochechos com clorexidina) } \\
\text { sempre que possível. O uso de tetracicliclinas é contra } \\
\text { indicado em crianças }<8 \text { anos. }\end{array}$ \\
\hline Líquen plano erosivo & $\begin{array}{l}\text { Corticóide tópico na menor dose possível, por, no máximo } \\
\text { duas semanas. }\end{array}$ \\
\hline
\end{tabular}

Q uadro 2 - Tratamento das principais complicações bucais do diabetes melito.

miocárdio (SO UZA et al., 2003). Em pacientes com descontrole metabólico, a indicação de anestésico com adrenalina é controversa. Alguns autores recomendam evitar uso de soluções com vasoconstrictores à base de adrenalina e noradrenalina, pois essas promovem a quebra 
de glicogênio em glicose, aumentando ainda mais os níveis de glicose circulante (SO U ZA et al., 2003; TÓFO LI et al., 2005). N esses casos, eles recomendam usar preparados sintéticos (felipressina), ou usar anestésicos sem vasoconstrictores (TÓFO LI et al., 2005). A lidocaina $2 \%$, mepivacaína $2 \%$, articaína $4 \%$ associados à adrenalina 1:100.000, prilocaína $3 \%$ com felipressina a $0,03 \mathrm{UI} / \mathrm{mL}$, ou mepivacaína 3\% (sem vasoconstrictor) são opções de anestésicos locais usados em pacientes com DM .

Procedimentos:

a) radiografias, exame físico e moldagens: podem ser feitos sem restrição;

b) exodontias, raspagem e cirurgias periodontais, endodontia, apicectomia, colocação de bandas ortodônticas, injeções anestésicas locais intraligamentares e limpeza profilática como sangramento: avaliar uso de antibioticoterapia (O RSO ; PAG N O N CELLI, 2002; SO U ZA et al., 2003; HORLIAN A et al., 2005);

c) implantes osteointegrados: contra-indicados nos $\mathrm{DM}$ descontrolado, devido à alteração na síntese do colágeno (SO UZA et al., 2003);

d) tratamento hospitalar: é prudente que seja realizado em consulta com 0 endocrinologista.

\section{Situações especiais}

a) Insuficiência renal: não administrar drogas excretadas por via renal (gentamicina, amicacina). Os antibióticos, analgésicos ou antiinflamatórios de escolha são os metabolizados pelo fígado. AIN ES devem ser usados com cautela, porque podem promover retenção de sódio e água e provocar sangramento gástrico (SAN CHES et al., 2004).

b) H ipoglicemia: 0 cirurgião-dentista deve estar preparado para eventuais emergências relacionadas ao DM durante 0 tratamento odontológico, e o paciente deve ser encorajado a comunicar qualquer sensação de mal-estar (M EALEY et al., 1999; SO UZA et al., 2003). Se o paciente desenvolver sintomatologia sugestiva de hipoglicemia, o procedimento deverá ser suspenso imediatamente, e o paciente abordado como descrito no tópico sobre complicações.

c) Complicações bucais: o Q uadro 2 sumariza as principais complicações bucais do DM e seu tratamento.

\section{M edidas preventivas}

Pacientes bem controlados deverão ser avaliados a cada 6 meses, e aqueles com descontrole metabólico mais freqüentemente. $\mathrm{N}$ as consultas odontológicas, eles serão informados quanto à técnica e à freqüência de escovação e do uso do fio dental, e receberem profilaxia para cárie e periodontite com aplicação de flúor e remoção de placas e cálculo. A entrega de foIhetos explicativos com informações quanto à manutenção da saúde oral e condições que o façam suspeitar de problemas dentários é útil para ajudar a fixar o conhecimento. Situações que devem motivar a consulta ao dentista são: gengiva vermelha, inchada, que sangre ou tenha pus, mau hálito inexplicável, dor ao mastigar, dor de dente, boca seca, candidíase e dentes móveis ou "bambos" com exceção da troca de dentição decídua.

Ficha clínica: os Quadros 3 e 4 sumarizam uma ficha clínica para consulta odontológica do paciente com diabetes.

\section{CONSIDERAÇÕES FINAIS}

O DM é considerado um grave proble ma de saúde pública, devido ao aumento de sua incidência. Por ser uma doença sistêmica, tem influência em todo o organismo, inclusive na cavidade oral, aumentando a susceptibilidade à xerostomia, hiposalivação, candidíase e doença periodontal. Essa predisposição é maior em pacientes mal controlados.

Por isso, é importante que o cirurgiãodentista faça parte da equipe multiprofissional que cuida dos pacientes com D M . C abe ao dentista conhecer melhor essa patologia e suas manifestações bucais, estando preparado, inclusive, para atuar em casos de hipoglicemia durante 0 tratamento. Pacientes bem controlados de- 


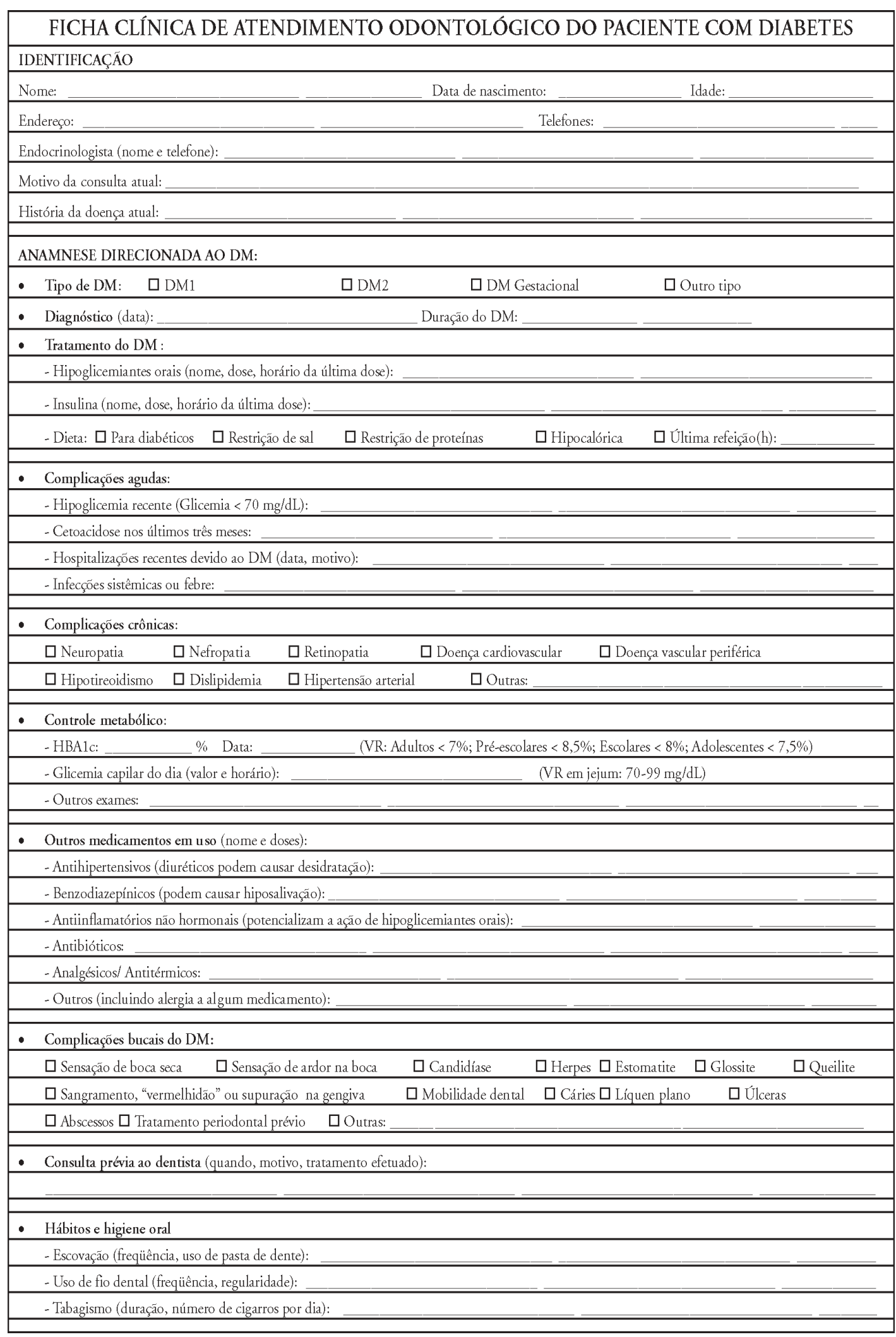

Q uadro 3 - Ficha de atendimento odontológico do pacientecom diabetes melito (Partel). 
EXAME FÍSICO:

- Pressão arterial (pacientes com nefropatia diabética):

- Glicemia capilar (antes do procedimento): (VR: $70-99 \mathrm{mg} / \mathrm{dL})$

- Exame das unidades dentárias:

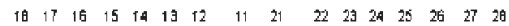

308000 90900000

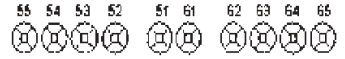

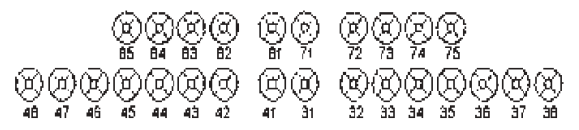

- Exame supragengival

$\square$ Gengivite $\square$ Hiperplasia gengival $\square$ Placa bacteriana $\square$ Cálculo dental $\square$ Cárie $\square$ Aparelho ortodôntico $\square$ Próteses mal adaptadas

$\square$ Restauraçóes com falta ou excesso de material $\quad \square$ Raizzes residuais $\quad \square$ Mobilidade das unidades dentais $\quad \square$ Queilite $\quad \square$ Herpes

$\square$ Candidíase $\square$ Estomatite $\square$ Glossite $\square$ Úlceras $\square$ Abscessos $\square$ Líquen plano

口 Fluxo salivar nắo estimulado:___ (Valor Normal $=0,3 \mathrm{a} 0,5 \mathrm{~mL} / \mathrm{min} ;$ Hiposalivaçăo $<0,1 \mathrm{~mL} / \mathrm{min}$ )

$\square$ Outras alteraçóes:

- Exame periodontal simplificado: Presença de sangramento a sondagem, maiores medidas de profundidade de sondagem, nível de inserçăo clínica e lesão de furca por dente.

\begin{tabular}{|c|c|c|c|c|c|c|}
\hline Dentes & Placa visivel & Cálculo & Profundidade de sondagem & Sangramento à sondagem & Nível de inserçáo & Lesáo de furca \\
\hline \multicolumn{7}{|l|}{18} \\
\hline \multicolumn{7}{|l|}{17} \\
\hline \multicolumn{7}{|l|}{16} \\
\hline \multicolumn{7}{|l|}{$15-55$} \\
\hline \multirow{2}{*}{\multicolumn{7}{|c|}{$14-54$}} \\
\hline & & & & & & \\
\hline \multicolumn{7}{|l|}{$13-53$} \\
\hline \multicolumn{7}{|l|}{$12-52$} \\
\hline \multicolumn{7}{|l|}{$11-51$} \\
\hline \multicolumn{7}{|l|}{$21-61$} \\
\hline \multicolumn{7}{|l|}{$22-62$} \\
\hline \multicolumn{7}{|l|}{$23-63$} \\
\hline \multirow{2}{*}{\multicolumn{7}{|c|}{$24-64$}} \\
\hline & & & & & & \\
\hline \multicolumn{7}{|l|}{$25-65$} \\
\hline \multicolumn{7}{|l|}{26} \\
\hline \multicolumn{7}{|l|}{27} \\
\hline \multicolumn{7}{|l|}{28} \\
\hline & & & & & & \\
\hline \multicolumn{7}{|l|}{38} \\
\hline \multicolumn{7}{|l|}{37} \\
\hline \multicolumn{7}{|l|}{36} \\
\hline \multicolumn{7}{|l|}{$35-75$} \\
\hline \multicolumn{7}{|l|}{$34-74$} \\
\hline \multirow{2}{*}{\multicolumn{7}{|c|}{$33-73$}} \\
\hline & & & & & & \\
\hline \multicolumn{7}{|l|}{$32-72$} \\
\hline \multicolumn{7}{|l|}{$31-71$} \\
\hline \multicolumn{7}{|l|}{$41-81$} \\
\hline \multicolumn{7}{|l|}{$42-82$} \\
\hline \multicolumn{7}{|l|}{$43-83$} \\
\hline & & & & & & \\
\hline $44-84$ & & & & & & \\
\hline $45-85$ & & & & & & \\
\hline 46 & & & & & & \\
\hline 47 & & & & & & \\
\hline 48 & & & & & & \\
\hline
\end{tabular}

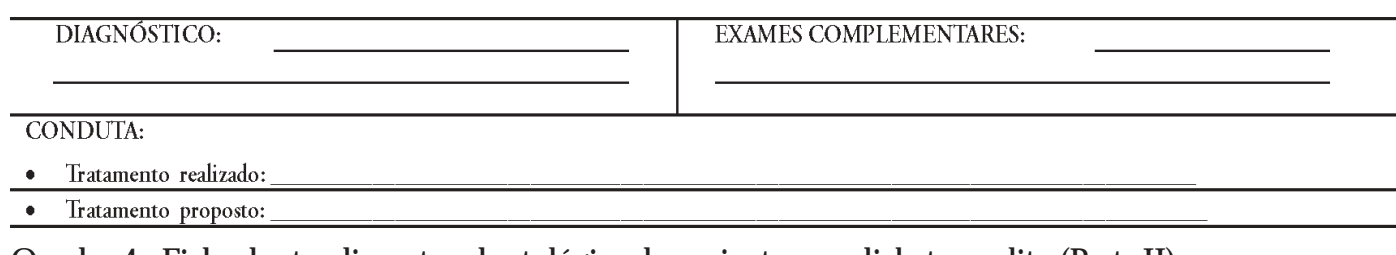

Q uadro 4 - Ficha deatendimento odontológico do paciente com diabetes melito (PartelI). 
vem ser tratados como não diabéticos, sem ne cessidade de cuidados especiais. Pacientes com descontrole metabólico deverão receber tratamento paliativo até a restauração do controle glicêmico. Pacientes com complicações crônicas serão tratados de acordo com suas limita- ções. Infelizmente, a literatura é pobre em estudos com boa qualidade metodológica para apoiar a maioria das condutas recomendadas, as quais se apóiam principalmente em conceitos teóricos.

\title{
D ental care of the patient with diabetes mellitus: clinical practice recommendations
}

\begin{abstract}
D iabetes mellitus (D M ) encompasses a group of metabolic diseases that have in common the presence of hyperglycemia. Besides its clinical complications, the poorly controlled DM is also associated with oral health disorders (e.g., periodontal disease, xerostomy, hypossalivation, susceptibility to infections, difficulty in healing). This paper aims to update the dental-surgeon on the current knowledge of DM and presents a protocol to guide the dental care of the diabetic patient. The literature review used the M ED LINE and LILACS database searching for articles published in the past ten years utilizing the following keywords. diabetes mellitus, dentistry, oral health and periodontal disease. The results are structured in topics. The first part presents updated information regarding the diagnosis, clinical, oral manifestations and treatment of the D M . The second part, proposes a clinical protocol in which are discussed, in a didactic manner (e.g., history, physical examination, subsidiary evaluation), the most common doubts related to the dental consult of the diabetic patient (e.g., antibiotic prophylaxis during procedures with high risk of bacteremia, use of antiinflammatory, sedatives and anesthetics with vasoconstrictors, definite versus palliative treatment, how to proceed in the event of hypoglycemia and hyperglycemia, when to suspect of a D M yet non diagnosed). At the end, a clinical sheet summarizes the most important aspects of this evaluation. Patients with well controlled diabetes can be treated similarly to non-diabetics for most routine dental needs. The dental practitioner, in communication with the assistant physician, plays an important role in the promotion and maintenance of the diabetic patient's quality of life.
\end{abstract}

Keywords: Diabetes mellitus-D entistry; O ral health; Periodontal disease.

\section{REFERÊNCIAS}

\section{ALBERTI, S. Citologia esfoliativa da mucosa bucal em pacientes diabéticos tipo II: morfologia e citomorfometria. 2002 Dissertação (M estrado)- Faculdade de 0 dontologia, Uni- versidade de São Paulo, Bauru, 2002.}

AMARAL, F.M.F.; RAMOS, P.G.A.; FERREIRA, S.R.G. Estudo da freqüência de cáries e fatores associados no diabetes mellitus tipo 1. Arq. Bras. Endocrinol. Metab., Rio de Janeiro, v.50, p.515-522, 2006.
AMERICAN DIABETES ASSOCIATION. $D$ iagnosis and classification of diabetes mellitus. Diabetes Care, Alexandria, v.27, p.S5-S10, 2004.

ANTUNES, S.F. et al. Diabetes mellitus e a doença periodontal. R. $\mathbf{O}$ donto. Ciênc., Porto Alegre, v.18, n.40, p.107-111, 2003.

CALLIARI, P.E.L. Diabetes mellitus tipo 1. In: ; GUERRA JÚ NIOR, G. (Ed). Endocrinologia pediátrica. São Paulo: Atheneu, 2004. p.121-128. 
CIANCOLA, L.J., et al. Prevalence of periodontal diseaes in insulin-dependent diabetes mellitus (juvenile diabetes). J. Am. D ent. Assoc., Chicago, v.104, p.653-660, 1982.

COSTA, C.C. et al. Estudo das manifestações bucais em crianças com diabetes e suas variáveis de correlação. Arq. Bras. Endocrinol. Metab., Rio de Janeiro, v.48, p.374-378, 2004.

DAJANI, A.S. et al. Prevention of bacterial endocarditis: recommendations by the American $\mathrm{H}$ eart Association. J. Am. D ent. Assoc., Chicago, v.277, p.1794-1801, 1997.

EISELEIN， L.; SCHWARTZ, H.J.; RUTLEDGE, J.C. The challenge of type 1 diabetes mellitus. ILAR J., Washington, DC, v.45, n.3, p.231-236, 2004.

FERREIRA, S.B.G.; VAN N U CCI, M.G.. $\mathrm{N}$ oções de diabetes para o não especialista. In: BRUNeTTE, C.M. (Ed). Periodontia médica: uma abordagem integrada. São Paulo: SEN AC, 2004. p.152-170.

FUNK, J.R. et al. Biochemical evaluation of early fracture healing in normal and diabetic rats. J. O rthop. Res., N ew York, v.18, p.126$132,2000$.

GREGHI, S.L.A. et al. Relação entre diabetes mellitus e doença periodontal. R. APCD, São Paulo, v.56, p.265-269, 2002.

H O RLIAN A, A.C.R.T. et al. Integração entre o cirurgião-dentista e o médico no atendimento dos diabéticos. R. Assoc. Paul. Cir. Dent., São Paulo, v.59. p.367, 2005.

KARJALAINEN, K.N.; KNUUTTILA, M.L. The onset of diabetes and poor metabolic control increase gingival bleeding in children and adolescents with insulin-dependent diabetes mellitus. J. Clin. Periodontol., Copenhagen, v.23, p.1060-1067, 1996.

KAWAMURA, J.Y. Avaliação clínica, radiográfica e imunohistoquímica da doença periodontal em pacientes portadores de diabetes mellitus tipo 1. 2002. D issertação (M estrado)-Faculdade de 0 dontologia, U niversidade de São Paulo, São Paulo, 2002.
M AN N A, T.D. et al. Diabetes mellitus na infância e adolescência. In: SETIAN, N . (Ed.) Endocrinologia pediátrica: aspectos físicos e metabólicos do recém-nascido ao adolescente. 2.ed. São Paulo: Sarvier, 2002. p.195-229.

MEALEY, B. et al. Diabetes and periodontal diseases. J. Periodontol., Chicago, v.70, p.935949, 1999.

M IRALLES, L.J. et al. Estudio clínico sobre la patología bucodentaria en el paciente diabético tipo 1. Med. 0 ral., Valencia, v.7, p.58-62, 2002.

MIST RO, F.Z. et al. Diabetes mellitus: revisão e considerações no tratamento odontológico. R. Paul. 0 dontol., São Paulo, v.25, p.15-18, 2003.

MOORE, P.A. et al. Type 1 diabetes mellitus, xerostomia, and salivary flow rates. 0 ral Surg. O ral Med. O ral Pathol. O ral Radiol. Endod., St. Louis, v.92, n.3, p.281-291, Sept. 2001.

NEVILLE, B.W. et al. M anifestações orais e doenças sistêmicas. In: Patologia oral e maxilofacial. 2.ed. Rio de Janeiro: Guanabara Koogan, p601, 2004.

N OVAES, A.B. et al. M anifestations of insulindependent diabetes mellitus in the periodontium of young Brazilian patients: a 10year follow-up study. J. Periodontol., Chicago, v.68, p.328-334, 1997.

ORSO, V.A.; PAGN ON CELLI, R.M .. O perfil do paciente diabético e 0 tratamento odontológico. R. $\mathbf{O}$ donto Ciênc., Porto Alegre, v.17, p.206-213, 2002.

PALMER, R.; SOORY, M. Fatores modificadores: diabetes, puberdade, gravidez e menopausa e tabagismo. In: LIN D HE, J. Tratado de periodontia clínica e implantologia oral. 4.ed. Rio de J aneiro: Guanabara Koogan, 2005. p.176-180.

SANCHES M.H. et al. Cuidados odontológicos em portadores de insuficiência renal crônica. R. Paul. $\mathbf{O}$ dontol., São Paulo, n.5, p.29-32, 2004. 
SANTANA D. et al. M anifestações orais em diabéticos metabolicamente descompensados. RG 0, Porto Alegre, v.50, p.23-29, 2002.

SHIP, J.A. Diabetes and oral health. J. Am. Dent. Assoc., Chicago, v.134, p.4S-10S, 2003.

SILVERSTEIN, J. et al. Care of children and adolescents with type 1 diabetes. D iabetes $C$ are, Alexandria, v.28, p.186-212, 2005.

SO UTHERLAND, J.H. et al. Commonality in chronic inflammatory diseases: periodontitis, diabetes, and coronary artery disease. Periodontol. 2000, Copenhagen, v.40, p.13043, 2006.

SO UZA, R.R. et al. 0 paciente odontológico portador de diabetes mellitus. Pesq. Bras. O dontopediatr. Clin. Integr., João Pessoa, v.3, p.71-77, 2003.

SPAN HEIMER, R.G.; U M PIERREZ, G.E.; ST UM PF, V. D ecreased collagen production in diabetic rats. Diabetes, Alexandria, v.37, p.371-376, 1987.

TEKELI, A. et al. Candida carriage and $C$ andida dubliniensis in oropharyngeal samples of type-1 diabetes mellitus pacients. M ycoses, Berlin, v.47, p.315-318, 2004.

TÓ FO LI, G.R. et al. Tratamento odontológico em pacientes com diabetes mellitus. R. Assoc. Paul. Cir. Dent., São Paulo, v.59, p.306-310, 2005.

VASCONCELOS, L. et al. Influência do diabetes melito na reparação óssea: revisão de literatura. R. Assoc. Paul. Cir. D ent., São Paulo, v.58, p.339-342, 2004.

VERN ILLO, A.T. D ental considerations for the treatment of patients with diabetes mellitus. J. Am. D ent. Assoc., Chicago, v.134, p.24S33S, 2003.

WEHBA, C.; RODRIGUES, A.S.; SOARES, F.P. Diabetes e doença periodontal: uma relação bidirecional. In: BRUNNETTE, C.M . Periodontia médica: uma abordagem integrada. São Paulo: SEN AC, 2004. p.173-195.

WYNN, R.L.; M EILLER, T.F. D rugs and dry mouth. Gen. Dent., Chicago, v.49, n.1, p.1012, 14, 2001. 